\title{
Gráficos estadísticos en libros de texto de Educación Primaria: un estudio comparativo entre España y Chile
}

\author{
Statistic graphs in primary education textbooks: a comparative study \\ between Spain and Chile
}

\author{
Danilo Díaz-Levicoy* \\ Carmen Batanero ${ }^{* *}$ \\ Pedro Arteaga ${ }^{* * *}$ \\ María M. Gea ${ }^{* * * *}$
}

\begin{abstract}
Resumen
En este artículo presentamos un estudio comparativo de los gráficos estadísticos incluidos en libros de texto de Educación Primaria en Chile y España. Para cada país se han analizado las directrices curriculares y su implementación en 18 libros de texto, el tipo de actividad que se plantea, los gráficos involucrados, el nivel de lectura que se exige y la complejidad semiótica de la tarea. Los libros se adecúan, en general, a las directrices curriculares, aunque difieren en la cantidad de actividades. La principal actividad que se solicita en los libros españoles es leer el gráfico y la de calcular en los chilenos. Entre las similitudes destacan un predominio de los gráficos de barras, un nivel de lectura elemental y la representación de una distribución de datos en el gráfico.
\end{abstract}

Palabras-clave: Gráficos estadísticos. Libros de texto. Educación primaria.

\begin{abstract}
In this article, we present a comparative study of statistic graphs included in Spanish and Chilean primary education textbooks. For each country, we analyzed the curricular guidelines and also their implementation in 18 textbooks, the type of activity presented, the graphs involved, the demanded reading level, and the semiotic complexity were analyzed for the textbooks of both countries. The textbooks are in general suited to the curricular guidelines, even though they differ in the quantity of activities. The main activity present in Spanish textbooks is reading graphs, while calculating is predominant in the Chilean ones. Among the similarities we found a predominance of bar graphs, an elementary reading level, and the representation of a distribution in the graphs.
\end{abstract}

Keywords: Statistical graphs. Textbooks. Primary education.

\footnotetext{
* Máster en Didáctica de la Matemática por la Universidad de Granada (UGR), España. Doctorando en Ciencias de la Educación de la Universidad de Granada (UGR), Granada, España. Dirección postal: Campus de Cartuja, 18071, Granada, España. E-mail: dddiaz01@ hotmail.com

** Doctora en Matemática por la Universidad de Granada (UGR), España. Catedrática de la Universidad de Granada (UGR), Granada, España. Dirección postal: Campus de Cartuja, 18071, Granada, España. E-mail: batanero@ugr.es

*** Doctor en Didáctica de la Matemática por la Universidad de Granada (UGR), España. Profesor del Departamento de Didáctica de la Matemática de la Universidad de Granada (UGR), Granada, España. Dirección postal: Campus de Cartuja, 18071, Granada, España. E-mail: parteaga@ugr.es

Doctora en Ciencias de la Educación por la Universidad de Granada (UGR), España. Profesora del Departamento de Didáctica de la Matemática de la Universidad de Granada (UGR), Granada, España. Dirección postal: Campus de Cartuja, 18071, Granada, España. E-mail: mmgea@ugr.es
} 


\section{Introducción}

La relevancia de la estadística en la formación de los niños y jóvenes hace que diferentes autores reclamen una cultura estadística suficiente que permita al ciudadano interpretar y valorar la información estadística en los medios de comunicación y actividades profesionales (MURRAY; GAL, 2002; DEL PINO; ESTRELLA, 2012). Un elemento de esta cultura estadística son los gráficos estadísticos (ARTEAGA et al., 2011; BATANERO; ARTEAGA; RUIZ, 2010; DEL PINO; ESTRELLA, 2012; EUDAVE, 2009); su trabajo en el aula permite realizar una conexión entre diferentes disciplinas y la sociedad en la que estamos inmersos (ESPINEL, 2007).

Estas consideraciones han sido recogidas en diferentes documentos, como los estándares americanos (NATIONAL COUNCIL OF TEACHERS OF MATHEMATICS, 2000; COMMON CORE STATE STANDARDS INITIATIVE, 2010) y el Proyecto GAISE (FRANKLIN et al., 2005) que resaltan la importancia de la formación estadística mediante una introducción progresiva y con datos extraídos de contextos cercanos. En los países en los que desarrollamos nuestro estudio, los gráficos estadísticos se contemplan en el currículo de Educación Primaria del antiguo Ministerio de Educación y Ciencia (MEC, 2006) y el actual del Ministerio de Educación, Cultura y Deporte (MECD, 2014) en España, y Ministerio de Educación de Chile (MINEDUC, 2012).

En este trabajo nos interesamos por los libros de texto; que se consideran un recurso didáctico y pedagógico fundamental (PEREIRA; GONZÁLEZ, 2011), cumpliendo un rol articulador en el proceso de enseñanza y aprendizaje (DÍAZ-LEVICOY; ROA, 2014), reflejan las directrices curriculares (HERBEL, 2007) y favorecen la equidad y enriquecimiento en los sectores de vulnerabilidad socioeconómica y cultural (MINEDUC, 2008). Más concretamente, realizamos un estudio comparativo de las características de los gráficos estadísticos en libros de texto para la educación primaria en España y Chile, analizando la implementación de las directrices curriculares. En lo que sigue describimos los fundamentos del estudio, el método y resultados del trabajo, finalizando con algunas implicaciones para la práctica docente y formación de profesores.

\section{Fundamentos del estudio}

\subsection{Los gráficos estadísticos en el currículo español y chileno de Educación Primaria}


En España, la enseñanza de la matemática se organiza en cuatro bloques de contenido, dedicando el cuarto al Tratamiento de la información, azar y probabilidad. En dicho bloque encontramos los siguientes contenidos relacionados con gráficos estadísticos (MEC, 2006, p. 43101):

- Primer ciclo (6-7 años): descripción verbal, obtención de información cualitativa e interpretación de elementos de gráficos sencillos relativos a fenómenos cercanos.

- Segundo ciclo (8-9 años): interpretación y descripción verbal de gráficos sencillos relativos a fenómenos familiares. Disposición a la elaboración y presentación de gráficos y tablas de forma ordenada y clara.

- Tercer ciclo (10-11 años): distintas formas de representar la información. Tipos de gráficos estadísticos. Valoración de la importancia de analizar críticamente las informaciones que se presentan a través de gráficos estadísticos. Obtención y utilización de información para la realización de gráficos.

Al especificar los criterios de evaluación se mencionan los gráficos de barras y líneas. Actualmente, las directrices curriculares de la Educación Primaria española se están revisando y en el último documento publicado se hacen modificaciones leves a los contenidos anteriormente citados. Una diferencia a resaltar es que se incluyen, explícitamente, los gráficos de sectores (MECD, 2014).

La enseñanza primaria chilena comprende ocho niveles, comenzando, como la española, a los 6 años. En este estudio hemos considerado solo los seis primeros cursos, para poder realizar el estudio comparado y porque el MINEDUC tiene intención de reducir a 6 años de estudio la Educación Primaria. Entre los 5 ejes que propone el MINEDUC (2012), el de Datos y Probabilidades establece los siguientes objetivos de aprendizaje sobre gráficos:

- Primer año (MINEDUC, 2013a, p. 42): recolectar y registrar datos para responder preguntas estadísticas sobre sí mismo y el entorno, usando bloques, tablas de conteo y pictogramas. Construir, leer e interpretar pictogramas.

- Segundo año (MINEDUC, 2013b, p. 43): recolectar y registrar datos para responder preguntas estadísticas sobre juegos con monedas y dados, usando bloques y tablas de conteo y pictogramas. Registrar en tablas y gráficos de barra simple, resultados de juegos aleatorios con dados y monedas. Construir, leer e interpretar pictogramas con escala y gráficos de barra simple.

- Tercer año (MINEDUC, 2013c, p. 43-44): realizar encuestas, clasificar y organizar los datos obtenidos en tablas y visualizarlos en gráficos de barra. Construir, leer e 
interpretar pictogramas y gráficos de barra simple con escala, en base a información recolectada o dada. Representar datos usando diagramas de puntos.

- Cuarto año (MINEDUC, 2013d, p. 44): realizar encuestas, analizar los datos y comparar con los resultados de muestras aleatorias, usando tablas y gráficos. Realizar experimentos aleatorios lúdicos y cotidianos, y tabular y representar mediante gráficos de manera manual y/o con software educativo. Leer e interpretar pictogramas y gráficos de barra simple con escala, y comunicar sus conclusiones.

- Quinto año (MINEDUC, 2013e, p. 43): leer, interpretar y completar tablas, gráficos de barra simple y gráficos de línea y comunicar sus conclusiones. Utilizar diagramas de tallo y hojas para representar datos provenientes de muestras aleatorias.

- Sexto año (MINEDUC, 2013f, p. 43): comparar distribuciones de dos grupos, provenientes de muestras aleatorias, usando diagramas de puntos y de tallo y hojas. Leer e interpretar gráficos de barra doble y circulares y comunicar sus conclusiones.

En ambos países se observa un trabajo gradual con los gráficos estadísticos; aunque en el currículo chileno se explicita el tipo de gráfico que se debe trabajar en cada curso.

\subsection{Investigaciones sobre comprensión de gráficos estadísticos}

En este trabajo entendemos los gráficos estadísticos como representaciones de información (datos) que utilizan elementos geométricos (puntos, segmentos (longitudes), áreas etc.). La competencia gráfica implica el dominio de conocimientos estadísticos (variable, tipo de frecuencia etc.) y otros contenidos de la matemática escolar (porcentajes, fracciones, proporcionalidad, entre otros). A continuación describimos algunos trabajos que sirven de fundamento a nuestro estudio.

Curcio y cols. (CURCIO, 1989; FRIEL; CURCIO; BRIGHT, 2001) indican que es posible identificar los siguientes niveles de dificultad cuando se plantean preguntas que requieren la lectura de un gráfico:

- Leer los datos: es la lectura literal de la información mostrada en el gráfico estadístico.

- Leer dentro de los datos: es una lectura de información que aporta el gráfico estadístico y que no está explícitamente mostrada en él. Este nivel requiere aplicar 
procedimientos matemáticos sencillos (comparaciones, adiciones etc.) para obtenerla.

- Leer más allá de los datos: es cuando se solicita información que no está en el gráfico estadístico y no se puede deducir con operaciones o comparaciones directamente sino que requiere de un razonamiento por parte del sujeto. Consiste en predecir tendencias o valores usando la información representada en el gráfico.

- Leer detrás de los datos: es valorar críticamente la recogida de datos y la forma de recolección. Supone una reflexión del conocimiento matemático y del contexto.

Igualmente, la construcción de gráficos estadísticos involucra una actividad semiótica, pues en ella intervienen diferentes objetos matemáticos. En este sentido, Arteaga y cols. (ARTEAGA, 2008, 2011; BATANERO; ARTEAGA; RUIZ, 2010) definen los siguientes niveles de complejidad semiótica de un gráfico:

- Representación de datos individuales: gráfico que representa datos aislados, sin realizar una representación conjunta de ellos. No se utilizan los conceptos de variable ni distribución.

- Representación de un conjunto de datos, sin llegar a resumir su distribución: representación de cada dato de la distribución en un gráfico estadístico sin que estos sean agrupados.

- Representación de una distribución de datos: representación de una distribución de datos calculando las frecuencias asociadas.

- Representación de varias distribuciones sobre un mismo gráfico: representación de dos o más distribuciones de frecuencias en el mismo gráfico estadístico.

Algunos estudios previos han considerado los niveles de lectura y de complejidad de los gráficos estadísticos. Así, Méndez y Ortiz (2012) estudian la producción de tablas y gráficos estadísticos en 58 tesis de Licenciatura en Psicología Educativa de la Universidad Pedagógica Nacional de México. Sus resultados indican que los gráficos estadísticos, generalmente, representan la distribución de datos; predomina el nivel de lectura de leer dentro de los datos y una fuerte presencia de los gráficos de barras y de sectores.

Castellanos (2013) realiza un estudio de 45 problemas que incluyen gráficos y las tablas estadísticas propuestos en la Prueba SABER de Colombia para el área de matemática, en el periodo 2003-2009. Encuentra predominio de gráficos de barras; nivel de lectura de leer dentro los datos; representación de una distribución de datos y una tendencia a que el estudiante realice la actividad de lectura del gráfico, calcule a partir del mismo y que realice 
comparaciones de datos.

Mingorance (2014) analiza los gráficos y tablas estadísticas en las pruebas de diagnóstico andaluzas obligatorias para los niños de 10 años. Observa que los más frecuentes son los gráficos de barras, pero con presencia de todos los recomendados en el currículo. El nivel de competencia pedido es bajo según la actividad que se pide al estudiante (organizar, comprender e interpretar la información) y los contextos preferentes son los personales y sociales. Generalmente, se pide más de una actividad como leer, completar o traducir el gráfico y se utilizan los números naturales, aunque también los decimales o fracciones. Las magnitudes representadas son casi siempre discretas, pero aparecen ejemplos de magnitudes continuas como el peso o el tiempo.

\subsection{Investigaciones sobre libros de texto}

Las investigaciones sobre libros de texto son frecuentes en matemática, aunque en temas de estadística son más escasas. A continuación, describimos algunas investigaciones que conjugan la investigación sobre gráficos en libros de texto en diferentes niveles.

Lavalle, Micheli y Rubio (2006) analizaron el lenguaje gráfico que se utiliza para el tema de correlación y regresión en siete libros de secundaria en Argentina; se destaca el uso de diagramas de dispersión, útil para que el estudiante analice el grado y tipo de relación lineal. Esta situación es similar a la encontrada en el estudio de Gea et al. (2014), quienes analizaron el mismo tema en ocho libros españoles.

Gómez et al. (2013) estudian el lenguaje de la probabilidad en dos series de libros de texto españoles de Educación Primaria, analizando los gráficos que se usan. Como resultado se encuentra una gran diversidad de representaciones: tablas, gráficos de barras y sectores, pictogramas e histogramas.

\section{Metodología}

Para completar las investigaciones anteriores, el objetivo general de nuestro estudio fue analizar la presentación de los gráficos estadísticos en libros de texto para la Educación Primaria en España y Chile, con el propósito de encontrar resultados que sean relevantes para la mejora de la enseñanza y la formación de los profesores que han de enseñar este tema.

Este trabajo sigue una metodología de tipo cualitativa y descriptiva basada en el análisis de contenido, que estudia la naturaleza del discurso y que se utiliza para el análisis 
sistemático de documentos escritos (ZAPICO, 2006). La muestra es intencional y está formada por 18 libros de texto de cada país, que se encontraban vigentes durante el curso 2013-2014 y se detallan como anexo. Se han codificado como TC (textos chilenos) y TE (textos españoles). Se tomaron tres editoriales de gran tradición en cada país y se analizaron los libros de $1^{\circ}$ a $6^{\circ}$ en cada una (alumnado de 6 a 12 años). Se realizaron varias lecturas de los textos identificando todas aquellas actividades (ejercicios, ejemplos, definiciones, problemas) en que apareciese algún gráfico o se propusiese la realización de uno.

En la Tabla 1 observamos las actividades analizadas por curso y país en los textos: 215 en los textos españoles y 508 en los chilenos, en total 723. Observamos mayor número de actividades en los textos chilenos quizá porque las directrices chilenas explicitan, para cada nivel, el tipo de gráfico estadístico que se debe trabajar; mientras las españolas son más generales. En ambos países las actividades aparecen, principalmente, en los niveles de $3^{\circ}, 5^{\circ}$ y $6^{\circ}$; en Chile, el $3^{\circ}$ de primaria concentra la mayor cantidad de actividades (23,6\%), seguido por $6^{\circ}(22 \%)$ y $5^{\circ}(20,7 \%)$; en el caso de España, el $5^{\circ}$ curso contiene el $29,8 \%$ de las actividades, $6^{\circ}$ un $23,3 \%$ y $3^{\circ}$ un $20 \%$ de las actividades.

Tabla 1 - Frecuencia (F) y porcentaje (\%) de actividades analizadas según nivel educativo

\begin{tabular}{ccccccc}
\hline \multirow{2}{*}{ Nivel educativo } & \multicolumn{2}{c}{ Textos españoles } & \multicolumn{2}{c}{ Textos chilenos } & \multicolumn{2}{c}{ Total } \\
\cline { 2 - 7 } & $\mathbf{F}$ & $\mathbf{\%}$ & $\mathbf{F}$ & $\boldsymbol{\%}$ & $\mathbf{F}$ & $\mathbf{\%}$ \\
\hline $1^{\mathbf{0}}$ & 12 & 5,6 & 46 & 9,1 & 58 & 8 \\
$2^{\mathbf{0}}$ & 14 & 6,5 & 79 & 15,6 & 93 & 12,9 \\
$3^{\mathbf{0}}$ & 43 & 20 & 120 & 23,6 & 163 & 22,5 \\
$4^{\mathbf{0}}$ & 32 & 14,9 & 46 & 9,1 & 78 & 10,8 \\
$5^{\mathbf{0}}$ & 64 & 29,8 & 105 & 20,7 & 169 & 23,4 \\
$6^{\mathbf{0}}$ & 50 & 23,3 & 112 & 22 & 162 & 22,4 \\
\hline Total & 215 & \multicolumn{5}{c}{ Fuente: elaborado por los autores } \\
\hline
\end{tabular}

Las variables analizadas, cuyos resultados se exponen a continuación fueron las siguientes.

- Tipo de gráfico. Incluyendo los gráficos citados en las directrices curriculares (MEC, 2006; MECD, 2014; MINEDUC, 2012).

- Niveles de lectura. Considerando los aportes de Curcio y colaboradores (CURCIO, 1989; FRIEL, CURCIO Y BRIGHT, 2001).

- Niveles de complejidad semiótica. Considerando los trabajos de Arteaga y colaboradores (ARTEAGA, 2008, 2011; BATANERO; ARTEAGA; RUIZ, 2010).

- Tipo de actividad. Donde se han considerado las siguientes: leer, calcular, construir, ejemplo, completar, inventar problema, comparar/justificar, traducir y asignar variables; que se describen y ejemplifican en el apartado de resultados y análisis. 


\section{Resultados y análisis}

\subsection{Tipo de gráficos}

En las Tablas 2 y 3 mostramos los tipos de gráficos estadísticos que intervienen en las actividades analizadas por curso y país. A nivel general, observamos un predominio del gráfico de barras, que se presenta en alto porcentaje en todos los niveles en ambos países. Estos son adecuados para la introducción de los gráficos estadísticos a edades tempranas según Watson (2006). En segundo y tercer lugar, de los gráficos más frecuentes, observamos diferencias pues en los textos chilenos predominan los pictogramas y gráficos de puntos, mientras que en los españoles los gráficos de líneas y de sectores.

Por otra parte, en los dos primeros años de educación primaria, en los textos españoles se trabaja solamente gráficos de barras, mientras que en los chilenos se conjugan los de barras y pictogramas. En el tercer nivel vemos como se incluye el gráfico de puntos en ambos países, y el gráfico de líneas y pictograma en los libros españoles; aunque el predominio sigue siendo de los gráficos de barras.

Tabla 2 - Tipo de gráficos en textos españoles

\begin{tabular}{|c|c|c|c|c|c|c|c|c|c|c|c|c|c|c|}
\hline \multirow{2}{*}{$\begin{array}{l}\text { Tipo de } \\
\text { gráfico }\end{array}$} & \multicolumn{2}{|c|}{$1^{\circ}$} & \multicolumn{2}{|c|}{$2^{\circ}$} & \multicolumn{2}{|c|}{$3^{\circ}$} & \multicolumn{2}{|c|}{$4^{\circ}$} & \multicolumn{2}{|c|}{$5^{\circ}$} & \multicolumn{2}{|c|}{$6^{\circ}$} & \multicolumn{2}{|c|}{ Total } \\
\hline & $\mathbf{F}$ & $\%$ & $\mathbf{F}$ & $\%$ & $\mathbf{F}$ & $\%$ & $\mathbf{F}$ & $\%$ & $\mathbf{F}$ & $\%$ & $\mathbf{F}$ & $\%$ & $\mathbf{F}$ & $\%$ \\
\hline Barras & 12 & 100 & 14 & 100 & 26 & 60,5 & 10 & 31,3 & 26 & 40,6 & 11 & 22 & 99 & 46 \\
\hline Líneas & & & & & 10 & 23,3 & 10 & 31,3 & 18 & 28,1 & 7 & 14 & 45 & 20,9 \\
\hline Puntos & & & & & 3 & 7 & & & & & & & 3 & 1,4 \\
\hline Pictograma & & & & & 3 & 7 & 3 & 9,4 & 8 & 12,5 & 2 & 4 & 16 & 7,4 \\
\hline Sectores & & & & & & & 3 & 9,4 & 9 & 14,1 & 14 & 28 & 26 & 12,1 \\
\hline Histograma & & & & & & & & & & & 8 & 16 & 8 & 3,7 \\
\hline $\begin{array}{l}\text { Dos o más } \\
\text { gráficos }\end{array}$ & & & & & 1 & 2,3 & 2 & 6,3 & 3 & 4,7 & 6 & 12 & 12 & 5,6 \\
\hline Otros & & & & & & & 4 & 12,5 & & & 2 & 4 & 6 & 2,8 \\
\hline Total & 12 & 100 & 14 & 100 & 43 & 100 & 32 & 100 & 64 & 100 & 50 & 100 & 215 & 100 \\
\hline
\end{tabular}

Tabla 3 - Tipo de gráficos en textos chilenos

\begin{tabular}{|c|c|c|c|c|c|c|c|c|c|c|c|c|c|c|}
\hline \multirow{2}{*}{$\begin{array}{l}\text { Tipo de } \\
\text { gráfico }\end{array}$} & \multicolumn{2}{|c|}{$1^{0}$} & \multicolumn{2}{|c|}{$2^{\mathbf{0}}$} & \multicolumn{2}{|c|}{$3^{\mathbf{0}}$} & \multicolumn{2}{|c|}{$4^{0}$} & \multicolumn{2}{|c|}{$5^{0}$} & \multicolumn{2}{|c|}{$6^{\circ}$} & \multicolumn{2}{|c|}{ Total } \\
\hline & $\mathbf{F}$ & $\%$ & $\mathbf{F}$ & $\%$ & $\mathbf{F}$ & $\%$ & $\mathbf{F}$ & $\%$ & $\mathbf{F}$ & $\%$ & $\mathbf{F}$ & $\%$ & $\mathbf{F}$ & $\%$ \\
\hline Barras & 17 & 37 & 34 & 43 & 58 & 48,3 & 37 & 80,4 & 31 & 29,5 & 39 & 34,8 & 216 & 42,5 \\
\hline Líneas & & & & & & & & & 40 & 38,1 & 1 & 0,9 & 41 & 8,1 \\
\hline Puntos & & & & & 27 & 22,5 & & & & & 19 & 17 & 46 & 9,1 \\
\hline Pictograma & 27 & 58,7 & 44 & 55,7 & 31 & 25,8 & 9 & 19,6 & & & 4 & 3,6 & 115 & 22,6 \\
\hline Sectores & & & & & & & & & 1 & 1 & 24 & 21,4 & 25 & 4,9 \\
\hline Tallo y hojas & & & & & & & & & 20 & 19 & 21 & 18,8 & 41 & 8,1 \\
\hline $\begin{array}{l}\text { Dos o más } \\
\text { gráficos }\end{array}$ & 2 & 4,3 & 1 & 1,3 & 3 & 2,5 & & & 4 & 3,8 & 1 & 0,9 & 11 & 2,2 \\
\hline Otros & & & & & 1 & 0,8 & & & 9 & 8,6 & 3 & 2,7 & 13 & 2,6 \\
\hline Total & 46 & 100 & 79 & 100 & 120 & 100 & 46 & 100 & 105 & 100 & 112 & 100 & 508 & 100 \\
\hline
\end{tabular}


En el cuarto nivel los libros chilenos plantean actividades con gráficos de barras y pictogramas mientras que en los españoles, además de los anteriores, trabajan los de líneas y se introducen los gráficos de sectores y dispersión (incluido dentro de otros por encontrarse esporádicamente). Para el quinto curso, en los textos chilenos se introducen los gráficos de sectores, líneas, dispersión y diagrama de tallo y hojas, éste último no identificado en los textos españoles. En el sexto nivel se introducen las pirámides de población en ambos países, aunque en cantidades muy pequeñas (también agregado en otros), y en los textos españoles se plantean actividades donde intervienen histogramas.

Como resumen, observamos un cumplimiento de las directrices curriculares respecto a los tipos de gráficos. En los textos españoles los gráficos más frecuentes son los de barras, en todos los niveles, y los de líneas se trabajan desde el tercer curso. En los libros de texto chilenos el primer nivel se cumple con la inclusión de pictogramas (MINEDUC, 2013a) y agregando los de barras, que se establecen para el segundo nivel (MINEDUC, 2013b); en tercero se agregan los gráficos de punto (MINEDUC, 2013c); en el cuarto curso encontramos gráficos de barras y pictogramas (MINEDUC, 2013d); en el quinto año se siguen las indicaciones del MINEDUC (2013e) pues se introducen los gráficos de líneas, de sectores, de tallo y hojas, y los gráficos de dispersión que no se explicitan en este nivel; finalmente, en el sexto curso, se mantienen los gráficos de tallo y hojas, sectores y barras múltiples (incluidos en los gráficos de barras) (MINEDUC, 2013f).

\subsection{Nivel de lectura}

En este punto analizamos los niveles de lectura definidos por Curcio (1989) y Friel, Curcio y Bright (2001). El primer nivel, de leer los datos, lo encontramos ejemplificado en la Figura 1, donde se pide hacer una lectura literal de la información del gráfico, relacionando el tres del dado (eje $X$ ) con la cantidad de veces que salió en los lanzamientos (eje $Y$ ). 


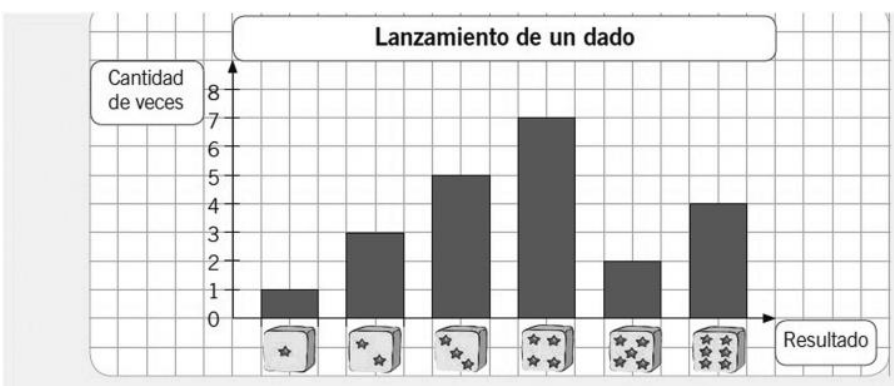

¿Cuántas veces se obtuvo 3 puntos al lanzar el dado?
A 1
B 3
C 5

Figura 1 - Ejemplo nivel 1 de lectura Fuente: TC8, p. 277

El segundo nivel, de leer dentro de los datos, se ejemplifica en la Figura 2, donde se pide al niño que realice una lectura de la frecuencia en cada categoría y haga un cálculo con ellas (diferencia entre la frecuencia de los niños que prefieren verano y primavera).

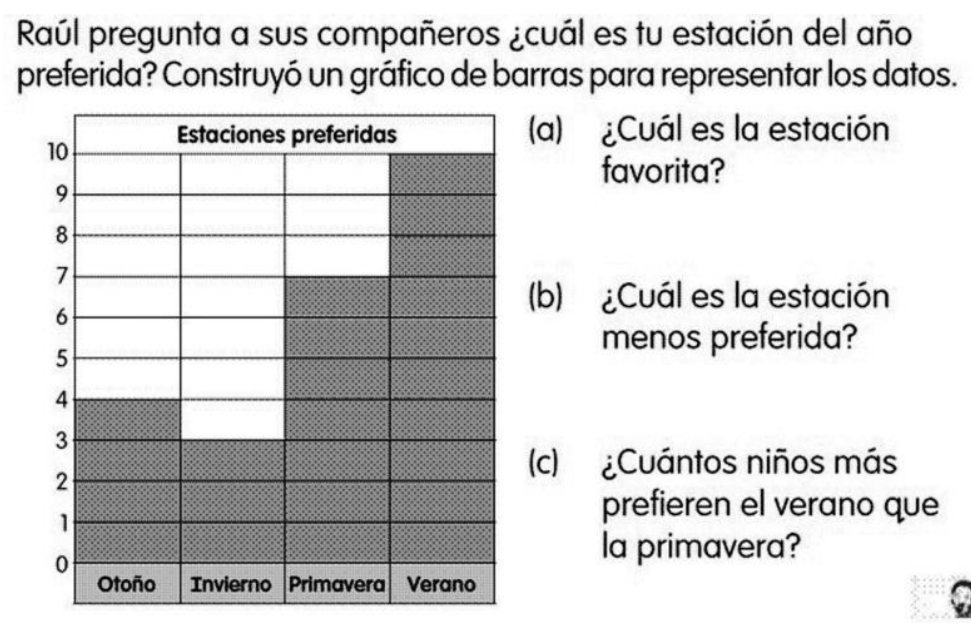

Figura 2 - Ejemplo nivel 2 de lectura Fuente: TC17, p. 97

Presentamos un ejemplo de nivel 3, de leer más allá de los datos, en la Figura 3, donde se muestra una actividad relacionada con la probabilidad, según los resultados (colores) al hacer girar una rueda. En ella se solicita predecir el color que saldrá en el próximo giro de acuerdo a la información que se muestra en el gráfico. Puesto que este dato no está en el gráfico, ni se obtiene mediante un procedimiento estandarizado de cálculos u operaciones, la tarea requiere una reflexión profunda de la construcción del gráfico y sus propiedades. 
José hizo girar 12 veces la rueda giratoria. Sus resultados se muestran en el siguiente diagrama de puntos. ¿Qué color predices que saldrá la próxima vez que José haga girar la rueda?
(A) Negro
(C) Azul
(B) Blanco
(D) Rojo

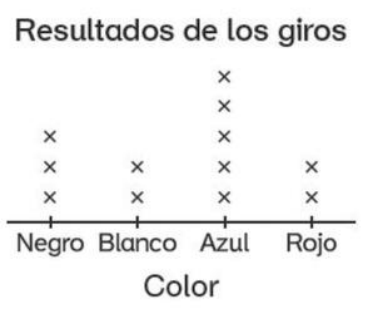

Figura 3 - Ejemplo nivel 3 de lectura Fuente: TC15, p. 32

Finalmente, el cuarto nivel de leer detrás de los datos se ejemplifica en la Figura 4. En esta actividad se solicita al niño que analice una conclusión obtenida de acuerdo al análisis del gráfico según la pregunta que se ha utilizado para recoger la información.

Grupo D Usa el gráfico de la derecha.

1. Pedro llegó a la conclusión de que la noche es el momento que más les gusta a las personas para ir al cine. ¿Es válida su conclusión?

2. La pregunta de la encuesta fue: “A mí me encanta ir al cine. ¿Cuándo prefieres ver películas tú?" Si el gráfico es confuso, explica por qué.

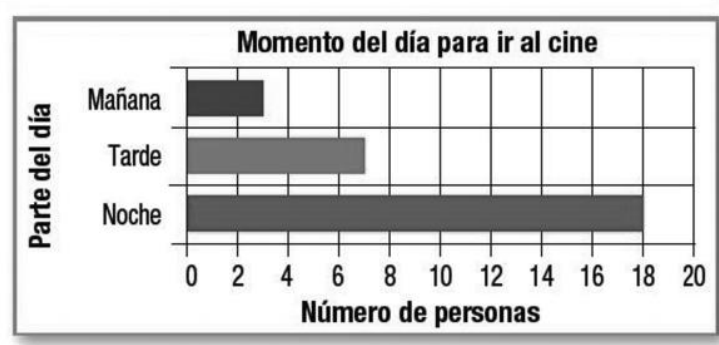

Figura 4 - Ejemplo nivel 4 de lectura Fuente: TC6, p. 262

En las Tablas 4 y 5 resumimos la distribución de las actividades según nivel de lectura. Observamos que el nivel más frecuente es el 1 (leer los datos) y el 2 (leer dentro de los datos) que agrupan el $90 \%$ de las actividades en ambos países. Se observan diferentes porcentuales, el $70,1 \%$ de las actividades con gráficos estadísticos de los textos chilenos exigen un nivel 2 de lectura siendo del 58,6\% en los textos españoles. Los niveles de lectura 1 y 2 se extienden por los seis niveles de primaria, siendo el $100 \%$ en los dos primeros cursos en ambos países. Los niveles 3 (leer más allá de los datos) y 4 (leer detrás de los datos) se presentan en forma ocasional a partir del tercer curso de primaria en Chile, y del cuarto curso en España. El tercer nivel de lectura se presenta en el cuarto, quinto y sexto curso de primaria en España, mientras que en Chile es en tercer y cuarto curso. En cuanto al cuarto nivel de lectura, los textos españoles solo lo incluyen en el quinto curso, siendo el doble de tareas las que se incluyen para este nivel en el caso chileno en los cursos tercero, cuarto, quinto y sexto de primaria. 
Tabla 4 - Nivel de lectura en textos españoles

\begin{tabular}{|c|c|c|c|c|c|c|c|c|c|c|c|c|c|c|}
\hline \multirow{2}{*}{$\begin{array}{l}\text { Nivel de } \\
\text { lectura }\end{array}$} & \multicolumn{2}{|c|}{$1^{0}$} & \multicolumn{2}{|c|}{$2^{\circ}$} & \multicolumn{2}{|c|}{$3^{\circ}$} & \multicolumn{2}{|c|}{$4^{0}$} & \multicolumn{2}{|c|}{$5^{\circ}$} & \multicolumn{2}{|c|}{$6^{0}$} & \multicolumn{2}{|c|}{ Total } \\
\hline & $\mathbf{F}$ & $\%$ & $\mathbf{F}$ & $\%$ & $\mathbf{F}$ & $\%$ & $\mathbf{F}$ & $\%$ & $\mathbf{F}$ & $\%$ & $\mathbf{F}$ & $\%$ & $\mathbf{F}$ & $\%$ \\
\hline 1 & 4 & 33,3 & 2 & 14,3 & 19 & 44,2 & 11 & 34,4 & 16 & 25 & 24 & 48 & 76 & 35,3 \\
\hline 2 & 8 & 66,7 & 12 & 85,7 & 24 & 55,8 & 20 & 62,5 & 40 & 62,5 & 22 & 44 & 126 & 58,6 \\
\hline 3 & & & & & & & 1 & 3,1 & 4 & 6,3 & 4 & 8 & 9 & 4,2 \\
\hline 4 & & & & & & & & & 4 & 6,3 & & & 4 & 1,9 \\
\hline Total & 12 & 100 & 14 & 100 & 43 & 100 & 32 & 100 & 64 & 100 & 50 & 100 & 215 & 100 \\
\hline
\end{tabular}

Tabla 5 - Nivel de lectura en textos chilenos

\begin{tabular}{|c|c|c|c|c|c|c|c|c|c|c|c|c|c|c|}
\hline \multirow{2}{*}{$\begin{array}{l}\text { Nivel de } \\
\text { lectura }\end{array}$} & \multicolumn{2}{|c|}{$1^{\circ}$} & \multicolumn{2}{|c|}{$2^{\circ}$} & \multicolumn{2}{|c|}{$3^{\circ}$} & \multicolumn{2}{|c|}{$4^{o}$} & \multicolumn{2}{|c|}{$5^{\circ}$} & \multicolumn{2}{|c|}{$6^{\circ}$} & \multicolumn{2}{|c|}{ Total } \\
\hline & $\mathbf{F}$ & $\%$ & $\mathbf{F}$ & $\%$ & $\mathbf{F}$ & $\%$ & $\mathbf{F}$ & $\%$ & F & $\%$ & F & $\%$ & F & $\%$ \\
\hline 1 & 20 & 43,5 & 25 & 31,6 & 38 & 31,7 & 4 & 8,7 & 22 & 21 & 5 & 4,5 & 114 & 22,4 \\
\hline 2 & 26 & 56,5 & 54 & 68,4 & 74 & 61,7 & 30 & 65,2 & 74 & 70,5 & 98 & 87,5 & 356 & 70,1 \\
\hline 3 & & & & & 7 & 5,8 & 4 & 8,7 & 5 & 4,8 & & & 16 & 3,1 \\
\hline 4 & & & & & 1 & 0,8 & 8 & 17,4 & 4 & 3,8 & 9 & 8 & 22 & 4,3 \\
\hline Total & 46 & 100 & 79 & 100 & 120 & 100 & 46 & 100 & 105 & 100 & 112 & 100 & 508 & 100 \\
\hline
\end{tabular}

Pese a la diferencia en cuanto a la cantidad de actividades de nivel 4 en los textos chilenos y españoles, esta diferencia no es significativa por tratarse de porcentaje muy reducido de ellas. Podemos decir que la lectura carece de una progresión armónica pues, a medida que se avanza en los cursos, se dificulta el logro de un análisis crítico de la información gráfica como se señala en diferentes documentos como el National Council of Teachers of Mathematics (2000) y las respectivas directrices curriculares de estos países (MEC, 2006; MINEDUC, 2012).

\subsection{Nivel de complejidad semiótica}

A continuación, ejemplificamos y resumimos los niveles semióticos, establecidos por Arteaga y cols. (ARTEAGA, 2008, 2011; BATANERO; ARTEAGA; RUIZ, 2010). Sólo se han encontrado tres ejemplos del primer nivel, de representación de datos individuales, siendo el nivel semiótico de menor frecuencia. Una de estas actividades se muestra en la Figura 5, donde se pide al estudiante determinar las coordenadas de un punto del gráfico de dispersión. La actividad es de nivel 1 porque sólo se representan datos aislados.
A. $(4,2)$
B. $(2,4)$
C. $(3,4)$
D. $(4,3)$

¿Cuáles son las plano cartesiano?

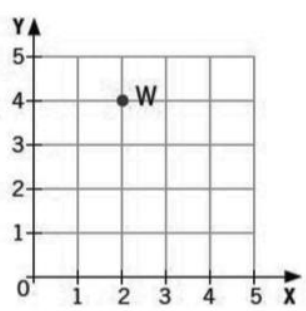

Figura 5 - Ejemplo nivel 1 de complejidad semiótica

Fuente: TC11, p. 303 
El segundo nivel de complejidad (representación de un conjunto de datos, sin llegar a resumir su distribución) lo ejemplificamos en la actividad de la Figura 6, donde, mediante un gráfico de líneas, se muestra la variación de la temperatura durante una semana. En este gráfico no existe un cálculo de frecuencias, ya que se representa la temperatura de cada día (dato a dato); sin embargo, el alumno ha de considerar la idea de variable, por lo que no se incluye en el nivel 1.

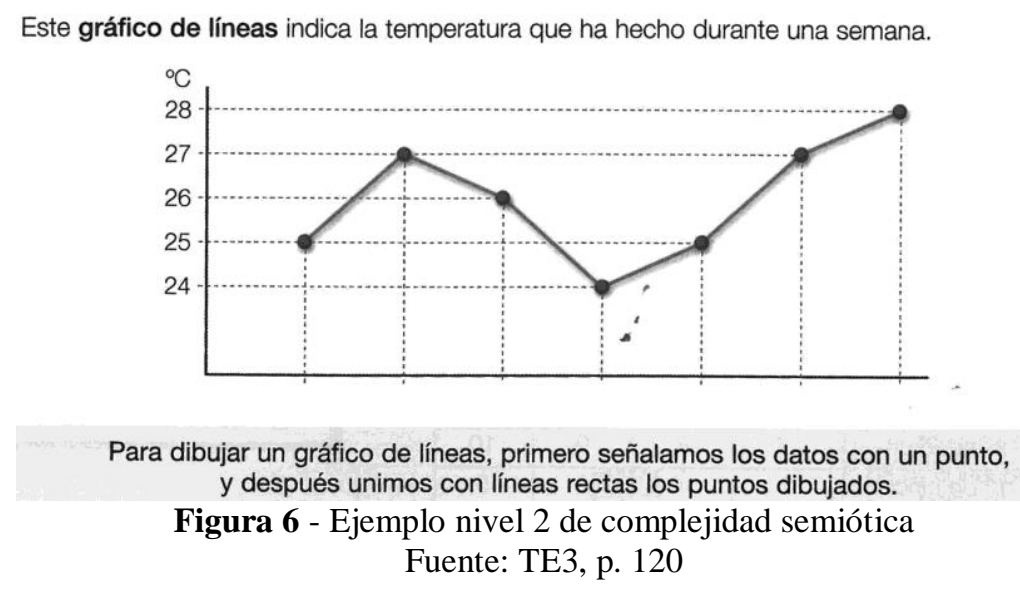

El nivel 3 (representación de una distribución de datos) se ejemplifica en la Figura 1, donde se presenta un gráfico de barras que agrupa las frecuencias de los resultados al lanzar un dado y grafica su distribución.

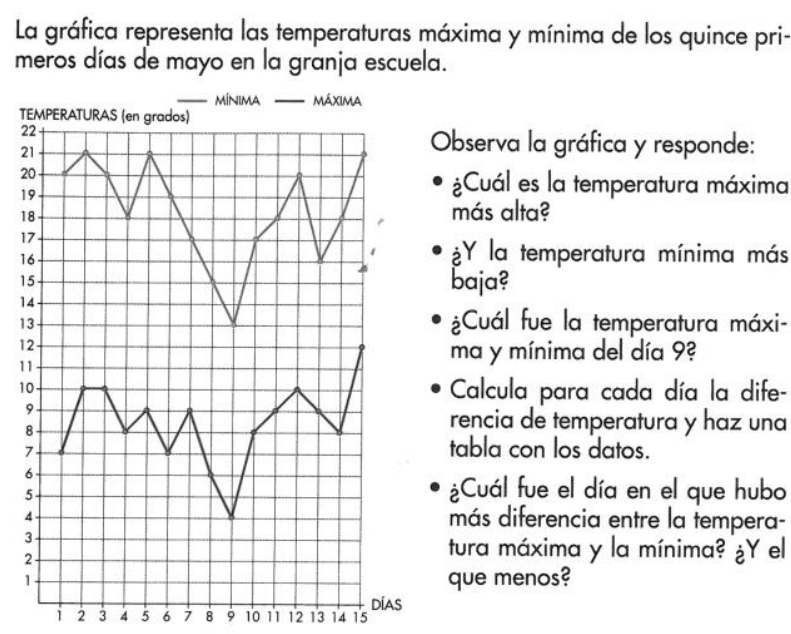

Figura 7 - Ejemplo nivel 4 de complejidad semiótica Fuente: TE10, p.169

Finalmente, el cuarto nivel de complejidad semiótica (representación de varias distribuciones sobre un mismo gráfico) se puede observar en la Figura 7, donde se representan las temperaturas máximas y mínimas en los primeros quince días de mayo en una granja escuela. La complejidad de este gráfico es mayor porque representa dos conjuntos de datos simultáneamente.

En las actividades analizadas (Tablas 6 y 7 ) encontramos que el nivel 3 de 
representación de una distribución de datos es el de mayor frecuencia, en torno al $60 \%$, en ambos países. Ello quiere decir que, en general, en los textos se trabaja con gráficos estadísticos según el concepto de distribución y no sólo la idea de variables estadística (nivel 2) o con datos aislados, (nivel 1). En los textos españoles no existe una diferencia significativa entre las actividades de segundo y cuarto nivel de complejidad: el 22,3\% corresponden al nivel 4 (representación de varias distribuciones sobre un mismo gráfico) y un 19,1\% al nivel 2 (representación de un conjunto de datos, sin llegar a resumir su distribución). Por el contrario, en los textos chilenos el segundo nivel más frecuente es el 2 (27\% de tareas) y el nivel 4 solo se presenta en un 6,3\% de actividades. Ejemplos del nivel 1 solo se encuentran en los textos chilenos.

Tabla 6 - Nivel de complejidad en textos españoles

\begin{tabular}{|c|c|c|c|c|c|c|c|c|c|c|c|c|c|c|}
\hline \multirow{2}{*}{$\begin{array}{l}\text { Nivel de } \\
\text { semiótico }\end{array}$} & \multicolumn{2}{|c|}{$1^{\circ}$} & \multicolumn{2}{|c|}{$2^{\circ}$} & \multicolumn{2}{|c|}{$3^{\circ}$} & \multicolumn{2}{|c|}{$4^{\circ}$} & \multicolumn{2}{|c|}{$5^{\circ}$} & \multicolumn{2}{|c|}{$6^{0}$} & \multicolumn{2}{|c|}{ Total } \\
\hline & $\mathbf{F}$ & $\%$ & $\mathbf{F}$ & $\%$ & $\mathbf{F}$ & $\%$ & $\mathbf{F}$ & $\%$ & $\mathbf{F}$ & $\%$ & $\mathbf{F}$ & $\%$ & $\mathbf{F}$ & $\%$ \\
\hline 2 & 3 & 25 & 2 & 14,3 & 7 & 16,3 & 14 & 43,8 & 13 & 20,3 & 2 & 4 & 41 & 19,1 \\
\hline 3 & 9 & 75 & 10 & 71,4 & 33 & 76,7 & 11 & 34,4 & 30 & 46,9 & 33 & 66 & 126 & 58,6 \\
\hline 4 & & & 2 & 14,3 & 3 & 7 & 7 & 21,9 & 21 & 32,8 & 15 & 30 & 48 & 22,3 \\
\hline Total & 12 & 100 & 14 & 100 & 43 & 100 & 32 & 100 & 64 & 100 & 50 & 100 & 215 & 100 \\
\hline
\end{tabular}

Tabla 7 - Nivel de complejidad en textos chilenos

\begin{tabular}{|c|c|c|c|c|c|c|c|c|c|c|c|c|c|c|}
\hline \multirow{2}{*}{$\begin{array}{l}\text { Nivel de } \\
\text { semiótico }\end{array}$} & \multicolumn{2}{|c|}{$1^{\circ}$} & \multicolumn{2}{|c|}{$2^{\circ}$} & \multicolumn{2}{|c|}{$3^{\circ}$} & \multicolumn{2}{|c|}{$4^{\circ}$} & \multicolumn{2}{|c|}{$5^{\circ}$} & \multicolumn{2}{|c|}{$6^{\circ}$} & \multicolumn{2}{|c|}{ Total } \\
\hline & $\mathbf{F}$ & $\%$ & $\mathbf{F}$ & $\%$ & $\mathbf{F}$ & $\%$ & $\mathbf{F}$ & $\%$ & $\mathbf{F}$ & $\%$ & $\mathbf{F}$ & $\%$ & $\mathbf{F}$ & $\%$ \\
\hline 1 & & & & & & & & & 2 & 1,9 & 1 & 0,9 & 3 & 0,6 \\
\hline 2 & 19 & 41,3 & 25 & 31,6 & 35 & 29,2 & 9 & 19,6 & 34 & 32,4 & 15 & 13,4 & 137 & 27 \\
\hline 3 & 27 & 58,7 & 54 & 68,4 & 85 & 70,8 & 37 & 80,4 & 57 & 54,3 & 76 & 67,9 & 336 & 66,1 \\
\hline 4 & & & & & & & & & 12 & 11,4 & 20 & 18 & 32 & 6,3 \\
\hline Total & 46 & 100 & 79 & 100 & 120 & 100 & 46 & 100 & 105 & 100 & 112 & 100 & 508 & 100 \\
\hline
\end{tabular}

\subsection{Tipo de actividades}

En este último apartado vamos a exponer los resultados del estudio sobre las actividades que se piden al estudiante en relación al gráfico estadístico. De una actividad, en la que intervienen gráficos estadísticos, se pueden identificar más de un tipo de actividad (por ejemplo: construir y calcular); esta situación ha sido considerada por separado para realizar el análisis, es decir, que se ha contabilizado dos veces o más, según corresponda.

En la Figura 1 observamos un ejemplo de la actividad de leer, donde se pide una lectura literal del número de veces que ha salido 3 al lanzar el dado, es decir, leer la frecuencia. En este tipo de actividad se incluye la lectura del título, ejes y escala del gráfico.

La Figura 7 muestra un ejemplo de la actividad de calcular, donde el niño debe leer la información que se presenta en el gráfico y realizar cálculos a partir de ella (temperatura 
máxima y mínima). De hecho, el estudiante debe, entre otras cosas, obtener la diferencia entre la temperatura máxima y mínima de cada día, comparar temperaturas máximas para obtener la mayor y las mínimas para obtener la menor.

La actividad de construir se ejemplifica en la Figura 8, donde se pide realizar la construcción de un gráfico de puntos con la información proporcionada. Para ello, el estudiante debe recordar cómo se construye el diagrama de puntos: trazar los ejes, proponer una escala, etiquetar los valores y finalmente representar cada dato para formar el gráfico. Hacemos notar que en el análisis de libros de texto se han encontrado actividades que conjugan esta tarea con otras actividades como por ejemplo leer, calcular y justificar/comparar, entre otras.

\begin{tabular}{|c|c|c|c|c|c|c|c|c|c|c|c|c|c|}
\hline \multicolumn{14}{|c|}{ Medallas olímpicas ganadas por 27 países } \\
\hline 8 & 88 & 59 & 12 & 11 & 57 & 38 & 17 & 14 & 28 & 28 & 26 & 25 & 23 \\
\hline 18 & 8 & 29 & 34 & 14 & 17 & 13 & 13 & 58 & 12 & 97 & 10 & 9 & \\
\hline
\end{tabular}

Figura 8 - Ejemplo actividad de construir Fuente: TC6, p. 253

La actividad ejemplo se refiere a aquellos casos en los que los gráficos estadísticos se incluyen en una sección o parte del libro de texto que se sirve para aclarar o definir algún concepto o idea; así como la actividad que se muestra en la Figura 6, donde se expone como se construye un diagrama de líneas basado en las temperaturas registradas en una semana.

Un ejemplo de la actividad completar lo observamos en la Figura 9, donde se debe continuar la construcción de un gráfico estadístico de acuerdo a la información proporcionada en la tabla. Este tipo de actividad no es de ejemplificar, pues tiene un componente de construcción y tampoco se incluye en estas últimas pues ofrece indicaciones y facilita la construcción al estudiante mediante la ejemplificación de algunos datos ya graficados.

La actividad de inventar problema la mostramos en la Figura 10, donde el niño debe generar un contexto en el que los datos que se aportan tengan sentido y coherencia. Es una actividad poco frecuente en los libros de texto analizados. Conlleva interpretar el contexto y plantear preguntas razonables que puedan responderse con los datos que se proporcionan. 


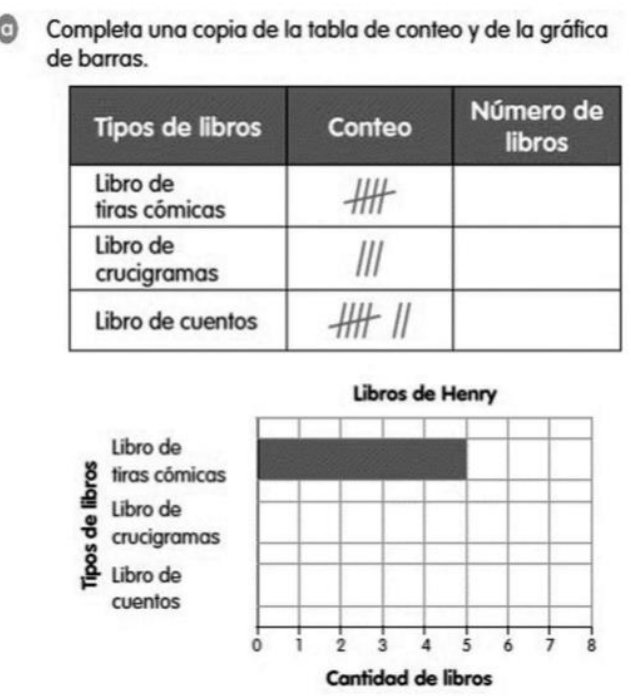

Figura 9 - Ejemplo actividad de completar Fuente: TC2, p. 198
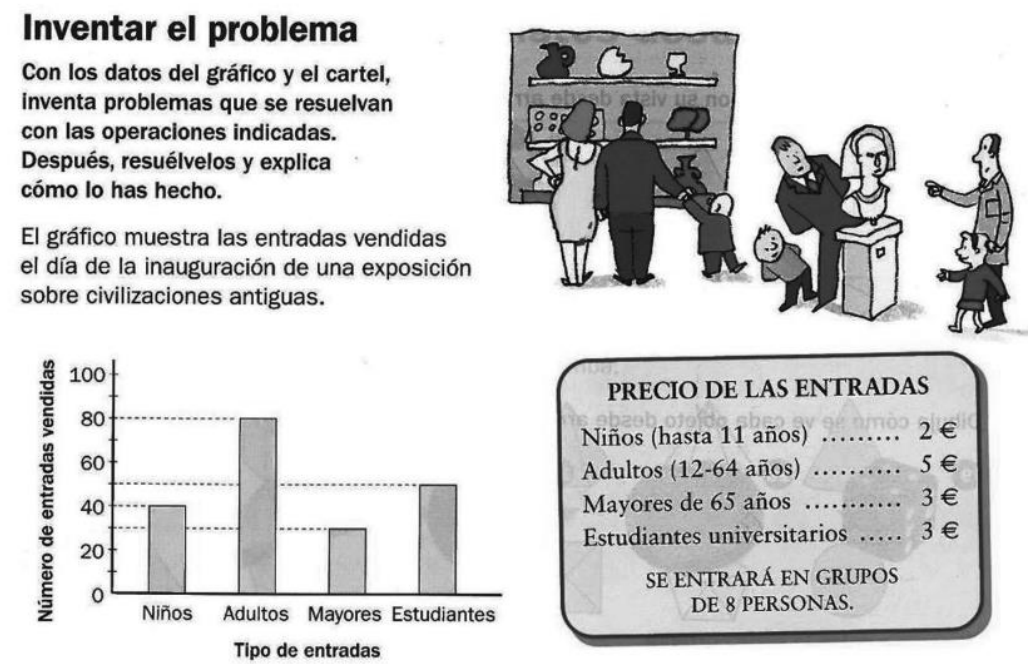

Figura 10 - Ejemplo actividad de inventar problema Fuente: TE15, p.194

En la Figura 11 se muestra un ejemplo de actividad de justificar/comparar, donde el estudiante debe construir un gráfico de tallo y hojas, señalar las ventajas de este tipo de gráfico frente al de barras, e indicar si se podría representar esta información en un gráfico de líneas. Es una actividad que requiere un conocimiento profundo sobre cada gráfico mencionado y también implica una capacidad argumentativa por parte del niño. 


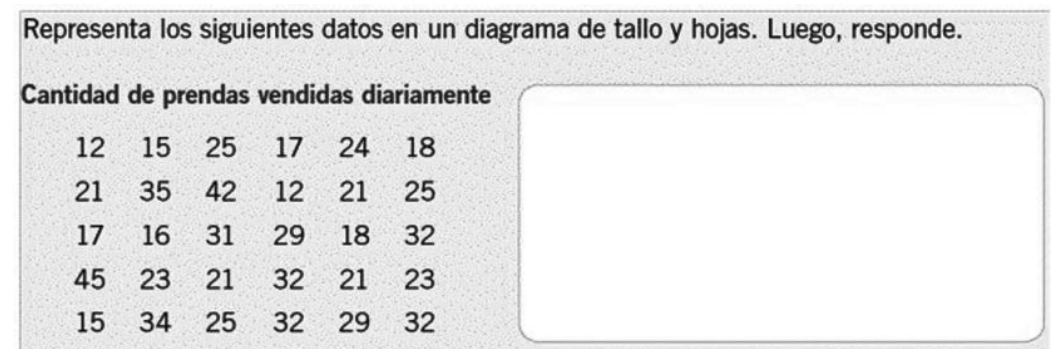

a. ¿Qué ventaja tiene en este caso utilizar un diagrama de tallo y hojas en vez de un gráfico de barras?

b. En este caso, ¿se podría haber representado la información en un gráfico de líneas? Explica.

Figura 11 - Ejemplo actividad de comparar/justificar Fuente: TC11, p. 277

La actividad de traducir consiste en pasar la información de un gráfico a una tabla $\mathrm{u}$ otro gráfico estadístico. Un ejemplo de esta actividad se muestra en la Figura 12, en la que se debe construir un gráfico de líneas con la información proporcionada en el gráfico de barras dobles. Esta actividad la consideramos compleja porque requiere que el niño haga un cambio de representación gráfica (leer la información del primer gráfico y construir el segundo) exigiendo un conocimiento de la lectura y construcción de ambos gráficos.

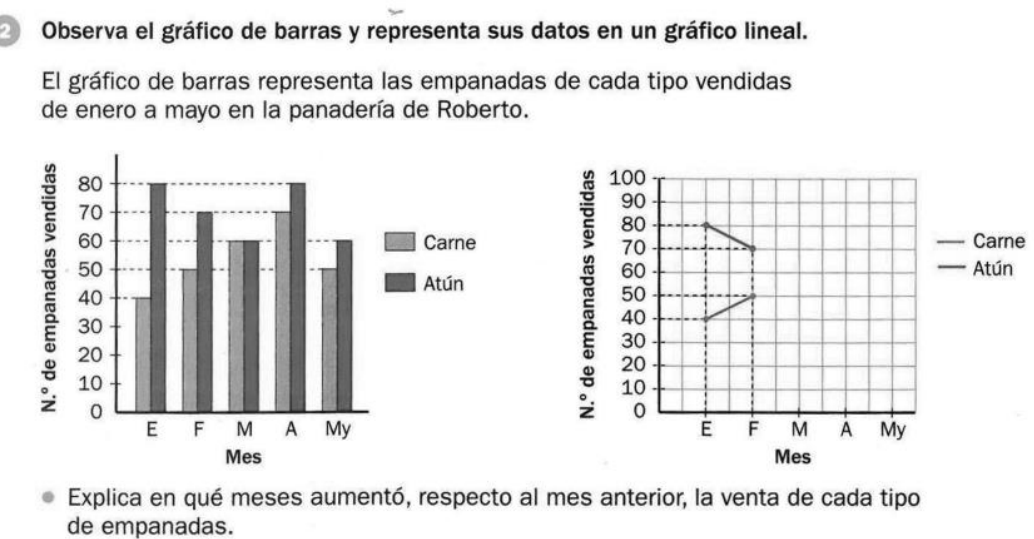

Figura 12 - Ejemplo actividad de traducir Fuente: TE17, p.128

En la Figura 13 presentamos un ejemplo de la actividad asignar variables, donde se pide al estudiante que mencione dos situaciones en que las gráficas tengan sentido, por ejemplo, consumo de electricidad en una casa y cantidad de agua en un estanque. Se trata de una actividad compleja, que solo se ha encontrado en los textos españoles. 
39 Inventa a qué situación puede referirse cada una de estas gráficas.

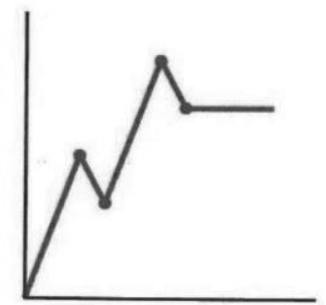

(1)

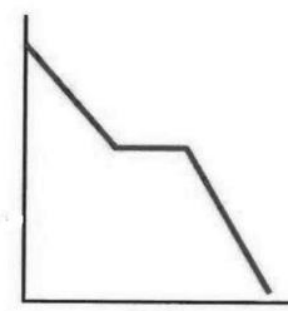

(2)

Figura 13 - Ejemplo actividad de asignar variable Fuente: TE5, p.120

En las Tablas 8 y 9 resumimos el tipo de actividades que se piden a los estudiantes en los textos analizados. En esta variable observamos diferencias en los dos países. En los libros de texto de España existe un predominio de la actividad de leer $(49,8 \%)$, construir $(27,9 \%)$ y ejemplo (16,7\%); además, estas actividades, con excepción de los ejemplos, se han identificado en todos los niveles de primaria española. En los libros de texto de Chile se observa un predominio de las actividades calcular (52,2\%), construir (24,8\%) y ejemplo $(12,6 \%)$, presentes en todos los niveles de primaria chilenos. Puesto que calcular implica una lectura previa, deducimos que los textos chilenos proponen, en general, actividades más completas que los españoles al añadir el cálculo a la lectura. El porcentaje de construcción de gráficos es similar en ambos países.

Las actividades de inventar problema y completar se presentan en pequeñas proporciones en los libros de texto de ambos países. Las actividades de justificar/comparar son mucho menores en los textos españoles que en los chilenos y ocurre lo contrario en las actividades de traducir.

Tabla 8 - Tipo de actividad en textos españoles

\begin{tabular}{|c|c|c|c|c|c|c|c|c|c|c|c|c|c|c|}
\hline \multirow[t]{2}{*}{ Tipo de actividad } & \multicolumn{2}{|c|}{$\begin{array}{c}\mathbf{1}^{\mathbf{0}} \\
(\mathrm{n}=12)\end{array}$} & \multicolumn{2}{|c|}{$\begin{array}{c}\mathbf{2}^{\mathbf{0}} \\
(\mathrm{n}=14)\end{array}$} & \multicolumn{2}{|c|}{$\begin{array}{c}\mathbf{3}^{\mathbf{0}} \\
(\mathrm{n}=43)\end{array}$} & \multicolumn{2}{|c|}{$\begin{array}{c}\mathbf{4}^{\mathbf{0}} \\
(\mathrm{n}=32)\end{array}$} & \multicolumn{2}{|c|}{$\begin{array}{c}\mathbf{5}^{\mathbf{0}} \\
(\mathrm{n}=64)\end{array}$} & \multicolumn{2}{|c|}{$\begin{array}{c}\mathbf{6}^{\mathbf{0}} \\
(\mathrm{n}=50)\end{array}$} & \multicolumn{2}{|c|}{$\begin{array}{c}\text { Total } \\
(\mathrm{n}=215)\end{array}$} \\
\hline & $\mathbf{F}$ & $\%$ & $\mathbf{F}$ & $\%$ & $\mathbf{F}$ & $\%$ & $\mathbf{F}$ & $\%$ & $\mathbf{F}$ & $\%$ & $\mathbf{F}$ & $\%$ & $\mathbf{F}$ & $\%$ \\
\hline Leer & 9 & 75 & 8 & 57,1 & 22 & 51,2 & 16 & 50 & 28 & 43,8 & 24 & 48 & 107 & 49,8 \\
\hline Completar & 1 & 8,3 & & & & & & & 1 & 1,6 & 1 & 2 & 3 & 1,4 \\
\hline Calcular & & & 1 & 7,1 & 3 & 7 & 3 & 9,4 & 9 & 14,1 & 3 & 6 & 19 & 8,8 \\
\hline Construir & 5 & 41,7 & 2 & 14,3 & 11 & 25,6 & 10 & 31,3 & 19 & 29,7 & 13 & 26 & 60 & 27,9 \\
\hline Traducir & & & 1 & 7,1 & 4 & 9,3 & 4 & 12,5 & 5 & 7,8 & 3 & 6 & 17 & 7,9 \\
\hline Ejemplo & & & 3 & 21,4 & 10 & 23,3 & 2 & 6,3 & 9 & 14,1 & 12 & 24 & 36 & 16,7 \\
\hline Justificar/comparar & & & & & & & 1 & 3,1 & 2 & 3,1 & & & 3 & 1,4 \\
\hline Asignar variables & & & & & & & & & 2 & 3,1 & & & 2 & 0,9 \\
\hline Inventar problema & & & & & 1 & 2,3 & & & & & & & 1 & 0,5 \\
\hline
\end{tabular}

Fuente: elaborado por los autores 
Tabla 9 - Tipo de actividad en textos chilenos

\begin{tabular}{|c|c|c|c|c|c|c|c|c|c|c|c|c|c|c|}
\hline \multirow[t]{2}{*}{ Tipo de actividad } & \multicolumn{2}{|c|}{$\begin{array}{c}\mathbf{1}^{\mathbf{0}} \\
(\mathrm{n}=46)\end{array}$} & \multicolumn{2}{|c|}{$\begin{array}{c}\mathbf{2}^{\mathbf{0}} \\
(\mathrm{n}=79)\end{array}$} & \multicolumn{2}{|c|}{$\begin{array}{c}\mathbf{3}^{\mathbf{0}} \\
(\mathrm{n}=120)\end{array}$} & \multicolumn{2}{|c|}{$\begin{array}{c}\mathbf{4}^{\mathbf{0}} \\
(n=46)\end{array}$} & \multicolumn{2}{|c|}{$\begin{array}{c}\mathbf{5}^{\mathbf{0}} \\
(n=105)\end{array}$} & \multicolumn{2}{|c|}{$\begin{array}{c}\mathbf{6}^{\mathbf{0}} \\
(\mathrm{n}=112)\end{array}$} & \multicolumn{2}{|c|}{$\begin{array}{c}\text { Total } \\
(n=508)\end{array}$} \\
\hline & $\mathbf{F}$ & $\%$ & $\mathbf{F}$ & $\%$ & $\mathbf{F}$ & $\%$ & $\mathbf{F}$ & $\%$ & $\mathbf{F}$ & $\%$ & $\mathbf{F}$ & $\%$ & $\mathbf{F}$ & $\%$ \\
\hline Leer & 4 & 8,7 & 6 & 7,6 & 10 & 8,3 & 1 & 2,2 & 6 & 5,7 & 3 & 2,7 & 30 & 5,9 \\
\hline Completar & 4 & 8,7 & 6 & 7,6 & 2 & 1,7 & 2 & 4,3 & & & & & 14 & 2,8 \\
\hline Calcular & 15 & 32,6 & 41 & 51,9 & 54 & 45 & 26 & 56,5 & 58 & 55,2 & 71 & 63,4 & 265 & 52,2 \\
\hline Construir & 11 & 23,9 & 17 & 21,5 & 37 & 30,8 & 4 & 8,7 & 27 & 25,7 & 30 & 26,8 & 126 & 24,8 \\
\hline Traducir & 4 & 8,7 & & & 1 & 0,8 & & & & & 2 & 1,8 & 7 & 1,4 \\
\hline Ejemplo & 6 & 13 & 12 & 15,2 & 15 & 12,5 & 8 & 17,4 & 12 & 11,4 & 11 & 9,8 & 64 & 12,6 \\
\hline Justificar/comparar & 4 & 8,7 & 1 & 1,3 & 4 & 3,3 & 8 & 17,4 & 11 & 10,5 & 9 & 8 & 37 & 7,3 \\
\hline Inventar problema & & & & & 3 & 2,5 & 1 & 2,2 & 1 & 1 & & & 5 & 1 \\
\hline
\end{tabular}

Fuente: elaborado por los autores

\section{Conclusiones}

Los resultados obtenidos nos permiten ver cómo se sugiere trabajar los gráficos estadísticos, desde qué curso, en qué progresión y la diversidad de actividades propuestas en libros de texto de Educación Primaria en España y Chile.

En primer lugar, nos permite establecer una gran diferencia en el número de actividades encontradas en los libros de texto chilenos y españoles, donde los primeros plantean más del doble de actividades. Con esto surge la interrogante de si los estudiantes podrán realizar todas las actividades propuestas o cómo selecciona el maestro las que se van a trabajar en el aula. En este sentido, las variables que se han definido en este trabajo podrían ayudar al profesor a seleccionar las más adecuadas para sus estudiantes.

Observamos, también, una gran variedad de gráficos, incluso sin que sean mencionados en las directrices curriculares. Así es que en España las directrices curriculares solo mencionan los gráficos de barras, líneas y sectores (MEC, 2006; MECD, 2014), y hemos observado la presencia también de gráficos de líneas, puntos y pictograma $\left(3^{\circ}\right)$, de dispersión $\left(4^{\circ}\right)$, sectores $\left(4^{\circ}\right.$ y $\left.5^{\circ}\right)$, pirámide e histograma $\left(6^{\circ}\right)$. En los libros de texto chilenos, se trabajan los gráficos indicados en las directrices curriculares (MINEDUC, 2013a, 2013b, 2013c, 2013d, 2013e, 2013f), aunque algunos de ellos se introducen antes de lo indicado (gráficos de barras en primero; sectores y dispersión en quinto; y pirámide de población en sexto curso). En los textos españoles no se observan actividades con diagramas de tallo y hojas, y en los chilenos no se encontraron actividades con histogramas.

Pese a las diferencias, el gráfico de barras es el más frecuente en ambos países, situación que se puede justificar porque es fácil de trabajar en edades tempranas, como lo menciona Watson (2006). Hay coincidencia con resultados de investigaciones previas (MÉNDEZ; ORTIZ, 2012; CASTELLANOS, 2013; MINGORANCE，2014), aunque en 
nuestro estudio se han identificado mayor diversidad de gráficos estadísticos.

En ambos países vemos un predominio del nivel de lectura 2 (leer dentro de los datos), que junto al nivel 1 (leer los datos) abarcan sobre el 90\% de las actividades. El predominio de este nivel de lectura coincide con los resultados encontrados por Castellanos (2013), donde se ha identificado este nivel en el $73 \%$ de las actividades analizadas; también en los de Méndez y Ortiz (2012) con un 67,25\% de los gráficos estudiados. Aunque nos parece adecuado, pensamos que en los últimos cursos de primaria debería existir mayor cantidad de gráficos de niveles de lectura 3 y 4 para lograr que los niños desarrollen una lectura crítica de la información a la que acceden.

El nivel semiótico de mayor frecuencia es el 3 (representación de una distribución de datos), en coincidencia con Castellanos (2013) y Méndez y Ortiz (2012) donde el 55,6\% y $67,24 \%$ de las actividades y gráficos, respectivamente, corresponden a esta nivel. Los libros de texto españoles presentan una distribución más homogénea del nivel de lectura, lo que puede favorecer la mejor comprensión de los gráficos estadísticos y sus elementos.

Encontramos diferencias en el tipo de actividad que se pide realizar. En los textos españoles predomina la actividad de lectura, al igual que en los estudios de Castellanos (2013) y Mingorance (2014), y en los chilenos la de calcular.

Pensamos que los resultados de esta investigación pueden ser de utilidad para profesores en ejercicio, principalmente de Chile y España, quienes deben tener claridad de las actividades que plantean a sus estudiantes, al tipo de gráfico que deben enseñar y si los libros de texto cumplen con los objetivos establecidos en las directrices curriculares. También sirven para los formadores de los profesores, quienes deben tener en cuenta los contenidos y ejercicios que estos tendrán que plantear a sus estudiantes para formarlos adecuadamente. Sugerimos que en dicha formación se trabaje con diversidad de gráficos y con cualquier nivel de lectura y semiótico. Sugerimos, finalmente, la necesidad de continuar este tipo de estudios en libros de texto de otros países, en otros niveles educativos o agregando otras variables.

Agradecimiento: Proyecto EDU2013-41141-P (MEC), Beca CONICYT PFCHA 72150306 y grupo FQM126 (Junta de Andalucía).

\section{Referencias}

ARTEAGA, P. Análisis de gráficos estadísticos elaborados en un proyecto de análisis de datos. 2008. 76f. Trabajo fin de Máster (Máster en Didáctica de la Matemática) - Departamento de Didáctica de la Matemática, Universidad de Granada, Granada, 2008. 
ARTEAGA, P. Evaluación de conocimientos sobre gráficos estadísticos y conocimientos didácticos de futuros profesores. 2011. 403f. Tesis (Doctorado en Didáctica de la Matemática) Departamento de Didáctica de la Matemática, Universidad de Granada, Granada, 2011.

ARTEAGA, P.; BATANERO, C.; CAÑADAS, G.; CONTRERAS, J. M. Las tablas y gráficos estadísticos como objetos culturales. Números, La Laguna, v. 76, p. 55-67, mar. 2011.

BATANERO, C.; ARTEAGA, P.; RUIZ, B. Análisis de la complejidad semiótica de los gráficos producidos por futuros profesores de educación primaria en una tarea de comparación de dos variables estadísticas. Enseñanza de las Ciencias, Barcelona, v. 28. n. 1, p. 141-154, 2010.

CASTELlANOS, M. Tablas y gráficos estadísticos en pruebas SABER - Colombia. 2013. 87f. Trabajo fin de Máster (Máster en Didáctica de la Matemática) - Departamento de Didáctica de la Matemática, Universidad de Granada, Granada, 2013.

COMMON CORE STATE STANDARDS INITIATIVE. Common Core State Standards for Mathematics. Washington: National Governors Association for Best Practices and the Council of Chief State School Officers, 2010.

CURCIO, F. R. Developing graph comprehension. Reston: NCTM, 1989.

DEL PINO, G.; ESTRELLA, S. Educación estadística: Relaciones con la matemática. Pensamiento Educativo. Revista de Investigación Educacional Latinoamericana, Santiago, v. 49, n. 1, p. 53-64, 2012.

DÍAZ-LEVICOY, D.; ROA, R. Análisis de actividades sobre probabilidad en libros de texto para un curso de básica chilena. Revista Chilena de Educación Científica, Santiago, v. 13. n. 1, p. 919,2014 .

ESPINEL, C. Construcción y razonamiento de gráficos estadísticos en la formación de profesores. Investigación en Educación Matemática, La Laguna, v. 11, p. 99-119, 2007.

EUDAVE, D. Niveles de comprensión de información y gráficas estadísticas en estudiantes de centros de educación básica para jóvenes y adultos de México. Educación Matemática, México, v. 21, n. 2, p. 5-37, 2009.

FRANKLIN, C.; KADER, G.; MEWBORN, D.; MORENO, J.; PECK, R.; PERRY, M.; SCHEAFFER, R. Guidelines for assessment and instruction in statistics education (GAISE) report: A Pre-K- 12 curriculum framework. Alexandria: American Statistical Association, 2005.

FRIEL, S.; CURCIO, F.; BRIGHT, G. Making sense of graphs: critical factors influencing comprehension and instructional implications. Journal for Research in Mathematics Education, Virginia, v. 32, n. 2, p. 124-158, 2001.

GEA, M. M.; BATANERO, C.; ARTEAGA, P.; CAÑADAS, G. R.; CONTRERAS, J. M. Análisis del lenguaje sobre la correlación y regresión en libros de texto de bachillerato. SUMA, Badalona, n. 76, p. $37-45,2014$

GÓMEZ, E.; ORTIZ, J. J.; BATANERO, C.; CONTRERAS, J. M. El lenguaje de probabilidad en los libros de texto de Educación Primaria. UNIÓN. Revista Iberoamericana de Educación Matemática, Sao Paulo, n. 35, p. 75-91, sep. 2013.

HERBEL, B. A. From intended curriculum to written curriculum: Examining the" voice" of a mathematics textbook. Journal for Research in Mathematics Education, Virginia, v. 38. n. 4, p. 344-369, 2007. 
LAVALLE, A. L.; MICHELI, E. B.; RUBIO, N. Análisis didáctico de regresión y correlación para la enseñanza media. Revista Latinoamericana de Investigación en Matemática Educativa, México, v. 9, n. 3, p. 383-406, nov. 2006.

MÉNDEZ, M.; ORTIZ, M. Construcción y lectura de gráficos y tablas estadísticas en tesis de la licenciatura en Psicología Educativa de la Universidad Pedagógica Nacional. 2012. 96f. Tesis (Licenciatura en Psicología Educativa) - Universidad Pedagógica Nacional Ajusco, México, 2012.

MINGORANCE, C. La estadística en las pruebas de diagnostico andaluzas. 2014. 23f. Trabajo Fin de Grado (Maestro de Educación Primaria) -Universidad de Granada, Granada, 2014.

MINISTERIO DE EDUCACIÓN, Chile. Política de Textos Escolares. Santiago: Autor, 2008.

MINISTERIO DE EDUCACIÓN, Chile. Matemática educación básica. Bases curriculares. Santiago: Unidad de Currículum y Evaluación, 2012.

MINISTERIO DE EDUCACIÓN, Chile. Matemática. Programa de estudio primer año básico. Santiago: Unidad de Currículum y Evaluación, 2013a.

MINISTERIO DE EDUCACIÓN, Chile. Matemática. Programa de estudio segundo año básico. Santiago: Unidad de Currículum y Evaluación, 2013b.

MINISTERIO DE EDUCACIÓN, Chile. Matemática. Programa de estudio tercer año básico. Santiago: Unidad de Currículum y Evaluación, 2013c.

MINISTERIO DE EDUCACIÓN, Chile. Matemática. Programa de estudio cuarto año básico. Santiago: Unidad de Currículum y Evaluación, 2013d.

MINISTERIO DE EDUCACIÓN, Chile. Matemática. Programa de estudio quinto año básico. Santiago: Unidad de Currículum y Evaluación, 2013e.

MINISTERIO DE EDUCACIÓN, Chile. Matemática. Programa de estudio sexto año básico. Santiago: Unidad de Currículum y Evaluación, 2013f.

MINISTERIO DE EDUCACIÓN Y CIENCIA, España. Real Decreto 1513/2006, de 7 de diciembre, por el que se establecen las enseñanzas mínimas correspondientes a la Educación primaria. Madrid: Boletín Oficial del Estado, nº 293, 2006.

MINISTERIO DE EDUCACIÓN, CULTURA Y DEPORTE, España. Real Decreto 126/2014, de 28 de febrero, por el que se establece el currículo básico de la Educación Primaria. Madrid: Boletín Oficial del Estado, $n^{\circ}$ 52, 2014.

MURRAY, S.; GAL, I. Preparing for diversity in statistics literacy: Institutional and educational implications. In: PHILLIPS, B. (Ed.). Proceedings of the Sixth International Conference on Teaching of Statistics. Ciudad del Cabo: IASE, 2002. CD ROM.

NATIONAL COUNCIL OF TEACHERS OF MATHEMATICS. Principles and standards for school mathematics. Reston: Autor, 2000.

PEREIRA, F.; GONZÁLEZ, G. Análisis descriptivo de Textos Escolares de Lenguaje y Comunicación. Literatura y Lingüística, Santiago, n. 24, p. 161-182, 2011.

WATSON, J. M. Statistical literacy at school: Growth and goals. Mahwah: Lawrence Erlbaum, 2006. 
ZAPICO, M. Interrogantes acerca de análisis de contenido y del discurso en los textos escolares. In: SEMINARIO INTERNACIONAL DE TEXTOS ESCOLARES, $1^{\circ}$, 2006, Santiago. Actas...

Santiago: Ministerio de Educación, 2006. p. 149-155.

Submetido em Maio de 2015. Aprovado em Outubro de 2015. 
Anexo - Libros de Texto Analizados

Libros de texto de Educación Primaria en España

TE1. FERRANDÍZ, B.; MONZÓ, A.; SANTAOLALLA, E. Matemáticas. 1 Primaria (Proyecto Trampolín). Madrid: SM, 2008.

TE2. FERRANDÍZ, B.; MONZÓ, A.; FERNÁNDEZ, B.; SANTAOLALLA, E. Matemáticas. 2 Primaria (Proyecto Trampolín). Madrid: SM, 2008.

TE3. PEÑA, M.; ARANZUBÍA, V.; SANTAOLALLA, E. Matemáticas $3^{\circ}$ (Proyecto Tirolina), Madrid: SM, 2008 (reedición 2011).

TE4. PEÑA, M.; ARANZUBÍA, V; SANTAOLALlA, E. Matemáticas $4^{\circ}$ (Proyecto Tirolina). Madrid: SM, 2008 (reedición 2011).

TE5. ARANZUBÍA, V.; SANTAOLALLA, E.; GÓMEZ, M.; PÉREZ, E. Matemáticas 5o (Proyecto Planeta Amigo). Madrid: SM, 2008.

TE6. ARANZUBÍA, V.; SANTAOLALLA, E.; ROLDÁN, J.; PÉREZ, E. Matemáticas $6^{\circ}$ (Proyecto Planeta Amigo). Madrid: SM, 2008.

TE7. FERRERO, L.; JIMÉNEZ, C.; MARTÍN, G. Matemáticas 1 (Salta a la vista). Madrid: Anaya, 2007.

TE8. FERRERO, L.; JIMÉNEZ, C.; MARTÍN, G. Matemáticas 2 (Salta a la vista). Madrid: Anaya, 2007.

TE9. FERRERO, L.; GAZTELU, I.; MARTÍN, P.; MARTÍNEZ, L. Matemáticas 3 (Proyecto Sol y Luna). Madrid: Anaya, 2001 (reedición 2004)

TE10. FERRERO, L.; GAZTELU, I.; MARTÍN, P.; MARTÍNEZ, L. Matemáticas 4 (Proyecto Sol y Luna). Madrid: Anaya, 2001 (reedición 2004)

TE11. FERRERO, L.; GAZTELU, I.; MARTÍN, P.; MARTÍNEZ, L. Matemáticas 5 (Proyecto Sol y Luna). Madrid: Anaya, 2001 (reedición 2004)

TE12. FERRERO, L.; GAZTELU, I.; MARTÍN, P.; MARTÍNEZ, L. Matemáticas 6 (Proyecto Sol y Luna). Madrid: Anaya, 2001 (reedición 2004)

TE13. GARÍN, M.; RODRÍGUEZ, M. Matemáticas 1 (Proyecto Un paso más). Madrid: Santillana, 2004 (reedición 2006)

TE14. GARCÍA, P.; GARÍN, M. Matemáticas 2 (Proyecto Un paso más). Madrid: Santillana, 2004 (reedición 2006)

TE15. ALMODÓVAR, J.A.; GARCÍA, F.; GARÍN, M.; GÓMEZ, R.; RODRÍGUEZ, M.; URIONDO, J.L. Matemáticas 3 (Proyecto Un paso más). Madrid: Santillana, 2005 (reedición 2006)

TE16. ALMODÓVAR, J.A.; GARCÍA, F.; GARÍN, M.; GÓMEZ, R.; RODRÍGUEZ, M.; URIONDO, J.L. Matemáticas 4 (Proyecto Un paso más). Madrid: Santillana, 2005.

TE17. ALMODÓVAR, J.A.; GARCÍA, F.; HERNÁNDEZ, J.; MORENO, R.; RODRÍGUEZ, M.; SERRANO, E. Matemáticas 5 (Proyecto Un paso más). Madrid: Santillana, 2006.

TE18. ALMODÓVAR, J.A.; GARCÍA, F.; HERNÁNDEZ， J.; MORENO, R.; RODRÍGUEZ, M.; SERRANO, E. Matemáticas 6 (Proyecto Un paso más). Madrid: Santillana, 2006.

Libros de texto de Educación Primaria en Chile

TC1. SALAZAR, R.; SPROVERA, M. Matemática $1^{\circ}$ Básico. Texto del Estudiante. Santiago: Fe y Alegría, 2014a.

TC2. HO KHEONG, F.; RAMAKRISHNAN, C.; PUI WAH, B. L.; CHOO, M. Mi Matemática. Texto del Estudiante $2^{\circ}$. Santiago: Marshall Cavendish Education, 2014a.

TC3. CHARLES, R.; CALDWELL, J.; CAVANAGH, M.; CHANCELLOR, D.; COPLEY, 
J.; CROWN, W.; FENNELL, F.; RAMIREZ, A.; SAMMONS, K.; SCHIELACK, J.; TATE, W.; VAN DE WALLE, J. Matemática $3^{\circ}$ Educación Básica. Texto del estudiante [Texto traducido y editado para el Ministerio de Educación]. Santiago: Pearson, 2014a.

TC4. ANDREWS, A.; DIXON, J.; NORWOOD, K.; ROBY, T.; SCHEER, J.; BENNETT, J.; LUCKIE, L.; NEWMAN, V.; SCARCELLA, R.; WRIGHT, D. Matemática $4^{\circ}$ Básico. Texto del estudiante [Texto traducido y editado para el Ministerio de Educación]. Santiago: Galileo, 2014a.

TC5. ANDREWS, A.; DIXON, J.; NORWOOD, K.; ROBY, T.; SCHEER, J.; BENNETT, J.; LUCKIE, L.; NEWMAN, V.; SCARCELLA, R.; WRIGHT, D. Matemática $5^{\circ}$ Básico. Texto del estudiante [Texto traducido y editado para el Ministerio de Educación]. Santiago: Galileo, 2014b.

TC6. ANDREWS, A.; DIXON, J.; NORWOOD, K.; ROBY, T.; SCHEER, J.; BENNETT, J.; LUCKIE, L.; NEWMAN, V.; SCARCELLA, R.; WRIGHT, D. Matemática 6 ${ }^{\circ}$ Básico. Texto del estudiante [Texto traducido y editado para el Ministerio de Educación]. Santiago: Galileo, 2014c.

TC7. BAEZA, A.; LOPEZ, F.; SANDOVAL, M.; URRA, A. Matemática $1^{\circ}$ Básico. Tomo II. Santiago: Santillana, 2013.

TC8. BAEZA, A.; BLAJTRACH, P.; KÜKENSHÖNER, C.; SANDOVAL, M. Matemática $2^{\mathbf{0}}$ Básico. Tomo II. Santiago: Santillana, 2013.

TC9. VÉLIZ, C. Matemática $3^{\circ}$ Básico. Tomo II. Santiago: Santillana, 2013.

TC10. BATARCE, Y.; CÁCERES, B.; KÜKENSHÖNER, C. (2013) Matemática $4^{\text {o }}$ Básico. Tomo II. Santiago: Santillana.

TC11. ÁVILA, J.; FUENZALIDA, C.; JIMÉNEZ, M.; RAMÍREZ, P. Matemática $\mathbf{5}^{\circ}$ Básico. Tomo II. Santiago: Santillana, 2013.

TC12. ÁVILA, J.; CASTRO, C.; MERINO, R.; RAMÍREZ, P. Matemática $6^{\circ}$ Básico. Tomo II. Santiago: Santillana, 2013.

TC13. SALAZAR, R.; SPROVERA, M. Matemática $1^{\circ}$ Básico. Cuaderno de ejercicios 4. Santiago: Fe y Alegría, 2014b.

TC14. HO KHEONG, F.; RAMAKRISHNAN, C.; PUI WAH, B. L.; CHOO, M. Mi Matemática. Cuaderno de trabajo ${ }^{\circ}$ B. Santiago: Marshall Cavendish Education, 2014b

TC15. CHARLES, R.; CALDWELL, J.; CAVANAGH, M.; CHANCELLOR, D.; COPLEY, J.; CROWN, W.; FENNELL, F.; RAMIREZ, A.; SAMMONS, K.; SCHIELACK, J.; TATE, W.; VAN DE WALLE, J. Matemática $3^{\circ}$ Educación Básica. Cuaderno de ejercicios 4 [Texto traducido y editado para el Ministerio de Educación]. Santiago: Pearson, 2014b.

TC16. ANDREWS, A.; DIXON, J.; NORWOOD, K.; ROBY, T.; SCHEER, J.; BENNETT, J.; LUCKIE, L.; NEWMAN, V.; SCARCELLA, R.; WRIGHT, D. Matemática $4^{\circ}$ Básico. Cuaderno de Práctica Tomo II [Texto traducido y editado para el Ministerio de Educación]. Santiago: Galileo, 2014d.

TC17. ANDREWS, A.; DIXON, J.; NORWOOD, K.; ROBY, T.; SCHEER, J.; BENNETT, J.; LUCKIE, L.; NEWMAN, V.; SCARCELLA, R.; WRIGHT, D. Matemática 5 ${ }^{\circ}$ Básico. Cuaderno de Práctica Tomo II [Texto traducido y editado para el Ministerio de Educación]. Santiago: Galileo, 2014e.

TC18. ANDREWS, A.; DIXON, J.; NORWOOD, K.; ROBY, T.; SCHEER, J.; BENNETT, J.; LUCKIE, L.; NEWMAN, V.; SCARCELLA, R.; WRIGHT, D. Matemática $6^{\circ}$ Básico. Cuaderno de Práctica Tomo II [Texto traducido y editado para el Ministerio de Educación]. Santiago: Galileo, $2014 \mathrm{f}$. 